$\mathbb{P}$ periodica polytechnica

\author{
Civil Engineering \\ $58 / 3(2014) 217,227$ \\ doi: $10.3311 /$ PPci.7472 \\ http://periodicapolytechnica.org/ci \\ Creative Commons Attribution (1) \\ RESEARCH ARTICLE
}

\section{Contribution to the platoon distribution analysis in steady-state traffic conditions}

\author{
Raffaele Mauro / Federico Branco / Marco Guerrieri
}

Received 2014-04-07, revised 2014-05-21, accepted 2014-07-04

\begin{abstract}
The traffic flow analysis and the relevant vehicle distribution ("free-moving" or "platooned" vehicles) on highway facilities at uninterrupted flow has always had fundamental importance in Highway Engineering, with special reference to topics like traffic operations, car accidents, road safety and air pollution emissions. In light of this, the study suggests a calculation algorithm as a random test generator to simulate a steady state traffic flow and to provide time headways. Thanks to the outcome produced by numerical simulations, we analysed platoon distributions within traffic flows in a steady-state regime and showed the results of numerical analyses carried out by traffic random process functions. The laws to determine "time headways" were obtained by the Pearson type III generalized distribution.
\end{abstract}

\section{Keywords}

Platoons - statistical model · steady-state - time headway. random function of traffic flow

\section{Raffaele Mauro}

Department of Civil, Environmental and Mechanical Engineering, University of Trento, via Calepina, 14 - I-38122 Trento, Italy

e-mail: raffaele.mauro@ing.unitn.it

\section{Federico Branco}

Consulting Engineer, Trento, Italy

e-mail: federico.branco@hotmail.it

\section{Marco Guerrieri}

Polytechnic School, University of Palermo, Piazza Marina, 61 - 90133 Palermo, Italy

e-mail: marco.guerrieri@tin.it

\section{Introduction}

The traffic flow composition and the relevant presence of vehicle platoons is particularly interesting in traffic study, and more generally in Highway Engineering, with reference to a plethora of theoretical and practical applications. For instance, as for "traffic operations" [1]-4], it is well known how the presence of platoons can influence breakdown probability [5, 6]. Moreover, platoon analyses turned out to be important also in the study of car accidents and road safety [7]. Such a very high practical interest accounts for the numerous models developed over the years. By way of an example, it is worth mentioning the research conducted by Baras et al. [8], which also considers facilities with interrupted flow - and the most recent studies by Ramezani et al. [9] and Jiang et al. [10]. Still today, therefore, the topic has a remarkable scientific and practical interest and deserves in-depth analysis.

At first, this article briefly describes the Pearson type III distribution which represents a time headway probability model (more specifically, a generalized mathematical model). The peculiarity of the Pearson type III distribution is its capacity to generate distribution families depending on the chosen model parameters, which can be suited to a plethora of types of traffic phenomena. In the course of this research, some of the above formulations were used to analyse a vehicle distribution within a traffic flow in steady-state conditions and notably to identify the presence and composition of vehicle platoons. In order to apply this analysis to a great number of observations, a specific algorithm was designed and calibrated according to empirical surveys, suitable to randomly simulate a traffic flow and to "identify" the essential characteristics of any present vehicle platoon. The algorithm was implemented to generate realizations of the random function $Q(t)$ (i.e. a traffic flow on a road cross-section in function of time $t$ ) starting from input data. The resulting random functions were properly studied and their main characteristics (i.e. non-random functions: mathematical hope and variance) were determined to confirm the steady state flow hypothesis assumed for the development of this study. 


\section{Time headway probability models}

By time headway probability model is meant a probability density function $f(t)$, or equivalently a distribution function $F(t)$, which describes the random variable $t$ "time interval between two instants when two consecutive vehicle passages occur in the observed road cross-section". In general, the distribution form can vary considerably in function of the stream flow rate in question.

As a matter of fact, when the flow rate has very low values (minimum interaction between vehicles), we can hypothesize that headways are distributed randomly.

When the flow increases, the interaction between vehicles increases as well and thus their headways are no more distributed randomly. At the extreme, that is when the flow rate is close to full capacity, nearly all vehicles interact one another and nearly all headways assume the same value.

If we consider the two extreme conditions of flow rate variation described above, intuitively we realize that for flow rate close to full capacities time headway distribution can be well represented by the normal distribution. On the contrary, for very low flow rates the statistical distribution which better represents such a situation is the negative exponential distribution [11].

However, the most frequent case - and consequently of greatest theoretical and practical interest - is represented by the intermediate situation comprised between the two extreme conditions described above where within the observed flow there are vehicles moving freely and other vehicles interacting with one another, thus developing a car-following process. In that case, time headway distribution can be shown by a generalized mathematical model which, as we will see, is valid also for one of the two extreme conditions previously described. In more detail, it is the Pearson type III distribution, whose probability density function is as follows:

$$
f(t)=\frac{\lambda}{\Gamma(K)}[\lambda(t-\alpha)]^{K-1} e^{-\lambda(t-\alpha)}
$$

where:

$\lambda$ is a parameter function of the mean headway and the successive $K$ and $\alpha$;

$K$ is a parameter, comprised between 0 and $+\infty$, which influences the distribution form;

$\alpha$ is a parameter, comprised between 0 and $+\infty$, which represents individual drivers' minimum headway (in seconds);

$\Gamma(K)$ is the Gamma function, equivalent to $(K-1)$ !.

Pearson type III distribution can be considered as a generalized mathematical model, in that it represents a family of distributions which can be determined by assigning appropriate values to coefficients $K$ and $\alpha$.

Table 1 shows the probability laws which can be obtained from the general form of the Pearson type III law. We note that in a case ( $K=1$ and $\alpha=1)$ we can also get the negative exponential law (apart from the shifted negative exponential law for
$K=1$ and $\alpha>0$ ), thought as the most appropriate in the extreme condition of a very low flow rate [11].

Tab. 1. Family of probability laws obtained by the Pearson type III law

\begin{tabular}{ccc}
\hline Model & $K$ value & $\alpha$ value \\
\hline Pearson type III & $\geq 0$ & $\geq 0$ \\
Gamma & $\geq 0$ & 0 \\
Erlang & $1,2,3, \ldots$ & 0 \\
Negative exponential & 1 & 0 \\
Shifted negative exponential & 1 & $>0$ \\
\hline
\end{tabular}

The family of distributions deriving from the complete form of the Pearson type III law can graphically be plotted on the plane $(K, \alpha)$ as illustrated in Fig. 1 .

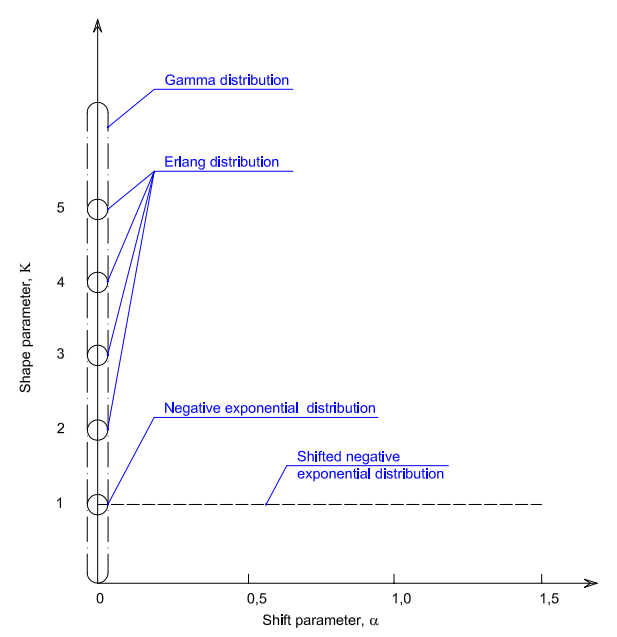

Fig. 1. Pearson type III distribution family on the plane $(K, \alpha)$

Every point on the plane of Fig. 11 and therefore each paired value $(K, \alpha)$ defines a single Pearson type III distribution. For the aims of this work, two models belonging to the Pearson type III distribution family, negative exponential and Erlang distribution laws, were chosen for the following reasons:

- simple closed-form expressions of cumulative probability function and its inverse (as detailed below);

- frequent use of models chosen in theoretical and practical applications;

- use of such models that $\alpha=0$, in that this parameter does not influence calculations developed in this work (please refer to paragraphs below for further details on the subject).

In detail, Table 2 shows the expressions of probability density function and probability cumulative function for the laws in this study, together with the estimation of $K$ and $\lambda$ parameters. 
We denote the distribution mean value with $\bar{t}$ and the standard deviation with s. By way of an example, Fig. 2 and Fig. 3 illustrate the graphs on probability distributions and probability density distributions for the laws in question.

In general, it is worth reminding that, if traffic counts are distributed according to a Poisson probability distribution, the time headway is an exponential variable; and also, if traffic counts are distributed with a generalized Poisson distribution, the time headway will be an Erlang probability function [12].

These functions were applied in the study of the platoon distribution in a traffic stream, as detailed in the later paragraphs of this article.

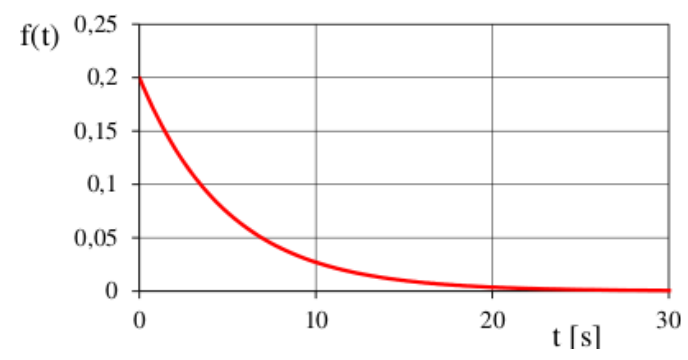

(a)

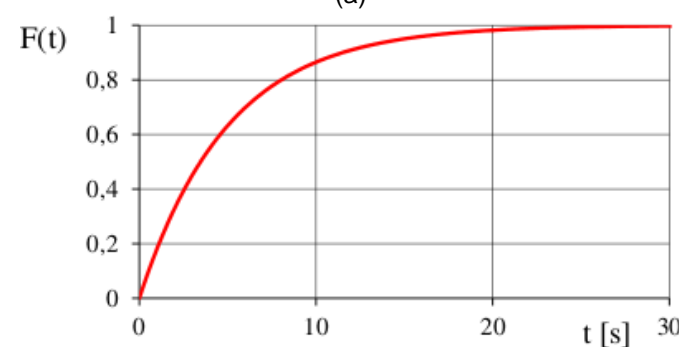

(b)

Fig. 2. Negative exponential: example of probability density function (a) and probability cumulative function (b), with $\bar{t}=5 \mathrm{sec}$

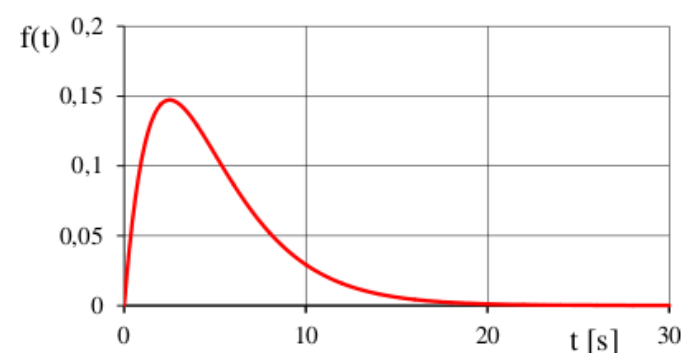

(a)

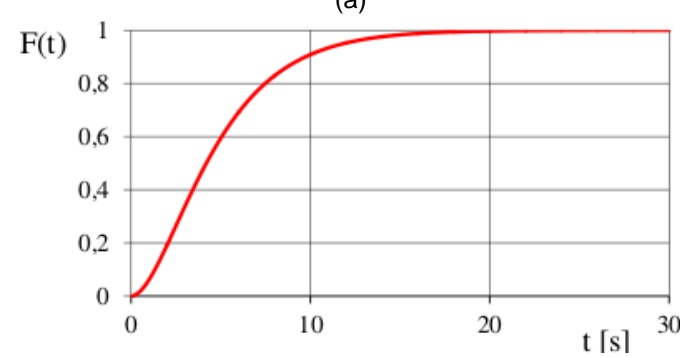

(b)

Fig. 3. Erlang: example of probability density function (a) and probability cumulative function (b), with $\bar{t}=5 \mathrm{sec}$ and $s=3.9 \mathrm{sec}$

\section{Platoon formation in a traffic stream}

Denoting the flow observation period with $T$, if we divide the period $T$ in " $n$ " equally long intervals, the flow is steady-state if the process mean, represented by the sequence of the random variables "numbers of vehicles driving along the road crosssection considered in the successive time intervals", is constant.

The constant of the mean in the process does not imply that the headway of two successive vehicle passages chosen randomly is equal to the mean headway of the steady state flow in question (or, in other words, it is equal to the reciprocal of the stream flow rate $1 / Q(t))$.

This is easily understood from Fig. 4, which shows a part of an empirical survey of time headways observed in a steady state traffic flow: on the abscissa axis there is the progressive number of the observed vehicle, on the ordinate axis there is the $n$-th time headway from the following vehicle, while the horizontal red segment shows the mean headway value seen in the entire observation interval $T$ (meant as the reciprocal of flow rate).

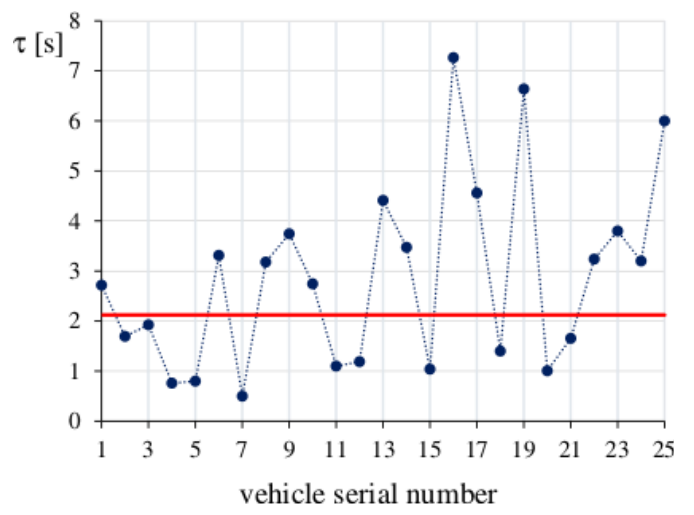

Fig. 4. Time headways observed in a steady state flow

In the example of Fig. 4, concerning 25 vehicles in transit, we observed headways ranging between 0.5 and 7 seconds; the mean headway value $\bar{t}$ in the entire observation interval $T$ is equal to about 2.1 seconds. This observation directly leads to point out that, as is well-known, in traffic flow there may be vehicles moving with long headways from the preceding and the following vehicles or, alternatively, vehicles which present reduced headways from either the preceding or the following vehicles or both. In light of this, a platoon can be defined [13] as the set of vehicles which all - with the only exception of the last in the file - have a lower time headway than the mean headway $\bar{t}$, whose number $N$ is higher than or equal to 2 and passing one behind the other on observational road cross-sections. With reference to the case shown in Fig. 4, we can note that:

- vehicles $(2,3,4,5,6)$ form a platoon. In fact, for vehicles 2 , 3,4 and 5 the headway from the successive vehicle is lower than the mean headway (observations are made below the red line) while vehicle 6 represents the last member of the platoon, given that the headway from the successive vehicle 7 turns out to be higher than the mean one;

- similarly, also sets $(7,8),(11,12,13),(15,16),(18,19)$ and 
Tab. 2. Probability density and cumulative functions for negative exponential and Erlang models

\begin{tabular}{cccc}
\hline Model & Parameter estimation & Probability density & Probability distribution \\
\hline Negative exponential & $\lambda=\frac{1}{\bar{t}}$ & $f(t)=\lambda e^{-\lambda t}$ & $F(t)=1-e^{-\lambda t}$ \\
Erlang & $K=\frac{\bar{t}}{s}$ & $f(t)=\frac{\lambda}{(K-1) !}[\lambda t]^{k-1} e^{-\lambda t}$ & $F(t)=1-e^{-\lambda t} \sum_{n=0}^{n=k-1} \frac{(\lambda t)^{n}}{n !}$ \\
\hline
\end{tabular}

\section{$(20,21,22)$ form platoons;}

- the remaining vehicles do not drive in platoons.

This study analyses some characteristics of platoon distributions within a steady-state traffic flow.

In order to analyse quite a sufficient number of data, which can hardly be collected from in situ surveys, an appropriate algorithm was created to randomly generate tests which simulate a steady state-regime flow and provide the generated vehicles with time headways.

The experiments carried out with this algorithm then allowed to study the platoon distribution.

\section{Random test generation algorithm}

For the aims of the research we created an algorithm to randomly generate tests which simulate a steady state-regime flow and provide the generated vehicles with time headway values. Such a tool offers the obvious advantage of randomly generating tests with reduced waste of resources (a common programming language was used) compared to measurement campaigns on operational roads.

It can be employed to study some phenomena linked to time headway distribution and, more in general, to traffic processes. The use of the algorithm led to the following conclusions which need, in any case, to be validated by empirical data from in situ surveys. In order to implement the algorithm, these are the input data:

- mean and variance of time headway distribution;

- time headway distribution law (Erlang and negative exponential distributions were applied);

- total length of the observational interval $T$ (assumed as follows: $T=900 \mathrm{sec}=15 \mathrm{~min}$ ).

The algorithm follows two essential steps:

- generation of a random number, between 0 and 1 , which represents the $F\left(t_{i}\right)$ value;

- calculation of $i$-th vehicle time headway by means of the inverse function of the probability distribution: $F^{-1}\left(t_{i}\right) \rightarrow t_{i}$.

The repetition of the procedure just described allows to determine the headways of all the vehicles forming the simulated traffic stream, of a total length $T$.

Therefore, the algorithm counts vehicle platoons and the vehicle number forming them as well as provide the values of other parameters specified below. The time headway in the model is obtained by the closed-form expressions of the inverse probability distribution functions. To this end, it is necessary to point out that:

- For the negative exponential distribution, the inverse function is as such:

$$
t=-\frac{\ln (1-F(t))}{\lambda}
$$

- In order to get the inverse Erlang distribution function, we initially applied Lambert $W$ function, defined by the expression:

$$
z=W(z) \cdot e^{W(z)}
$$

which is, in general, valid for any complex number $z$. In fact, if for simplicity we assume $K=2$ with simple algebraic steps applied to the probability distribution function, we obtain the following expression:

$$
e^{-\lambda t}(\lambda t+1)=1-F(t)
$$

Expression Eq. (3) can be solved in a closed form by applying the $W$ function as defined in Eq. (2). Such an algorithm minimizes calculus operations and the iteration number of computational processes which need to be performed by a computer, thus reducing the cycle execution times. Some tests were performed to verify the goodness and strength of the model. To this end, a sensitivity analysis was performed, carried out by varying input parameter values of the model within the respective variability ranges as usually observed in reality; the results obtained from the algorithm varied in a reasonable range. Moreover, a comparison was made between time headways observed in some measurement campaigns on operational roads [14-16 and those obtained from the model, in which simulation input data were the flows observed in measurement campaigns as well as other empirical data.

\section{Traffic flow simulations and result analysis}

As previously said the model was designed to generate tests for studying the number distribution of platooned vehicles within a traffic flow. We considered a constant flow rate (steady state flow hypothesis) in a 15-min observation period and got time headways for each test. The results of some tests are shown in Fig. 5 On these graphs the abscissa axis denotes the progressive number of the fictitious vehicle passage while the ordinate axis indicates the time headway value expressed in seconds; every point is then the graphical representation of the test conducted by random generation. 
The red horizontal line represents the headway mean value equal to the reciprocal of flow rate. Four scenarios with increasing flow rates $(720,1020,1320,1620 \mathrm{veh} / \mathrm{h})$ were analyzed and 10 random tests were generated for each scenario. In order to carry out this simulation we hypothesis that the headway distribution may be expressed by means of a negative exponential and/or an Erlang function. Eighty experiments were generated in all. Then, the number of vehicles driving in platoons as defined in paragraph 3 was analyzed for each test. The results are illustrated in the histogram of Fig. 6 where the columns represent the number of the platooned vehicles in question; the column height represents the absolute number of the observations made. Vehicles do not drive in platoons (first column) in around 50 times, while two-vehicle platoons are observed about 35 times; moreover, the number of the platooned vehicles is equal to 376. As a matter of fact, this is an evidence of a general nature, in the sense that in all the performed tests (including those with a lower flow) the number of free-moving vehicles was always inferior to the number of platooned vehicles. The main characteristics of the distributions obtained in the 80 simulated tests are summed up in the following tables. The characteristics are mean, variance and their ratio.

The tests are grouped in classes on the basis of the mean flow rate; Table 3 Table 4, Table 5and Table 6 show the results from both the time headway distributions (negative exponential and Erlang). In all cases the number mean of platoon-forming vehicles is maintained on values ranging between around 2 and 3 but no correlation is visible between the flow rate value and the mean itself. Similarly, the variance ranges between around 2 and 7 except for some isolated tests and the mean/variance ratio is always lower than 1.25. Fig. 7 and Fig. 8 show mean and variance values and their ratio in function of the flow rate $Q$ for time headway negative exponential and Erlang distributions respectively.

In Table 7 some results obtained by the comparison are shown (for the sake of simplicity, only five comparisons are shown). The flow rate mean value is the same between the results obtained from measurement campaigns on operational roads and those obtained from the model; in fact, the flow rate mean values are the input data for the model. It is also possible to see that results obtained from the application of the model are similar to those obtained from measurement campaigns: in particular, platoon number means show a deviation less than 7\%; variances show bigger deviations (in almost all cases less than 16\%).

However, the comparison of the parameters particularly interesting for the purpose of this study (such as flow mean and variance, platoon number mean and variance) pointed out deviations compatible with the random nature of the processes in question.

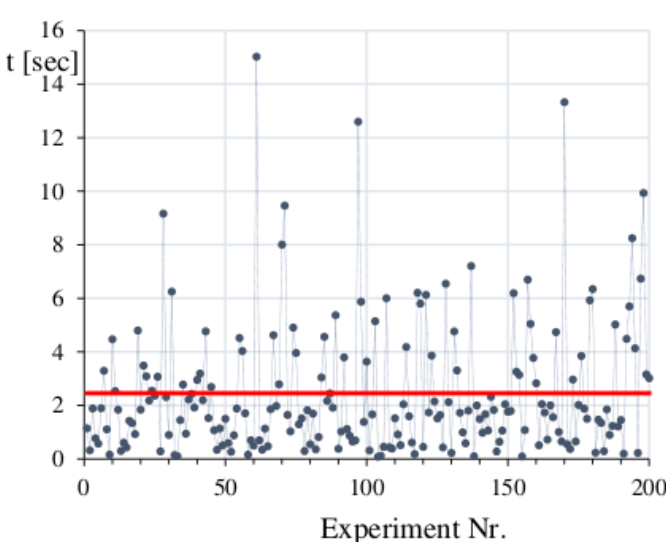

(a)

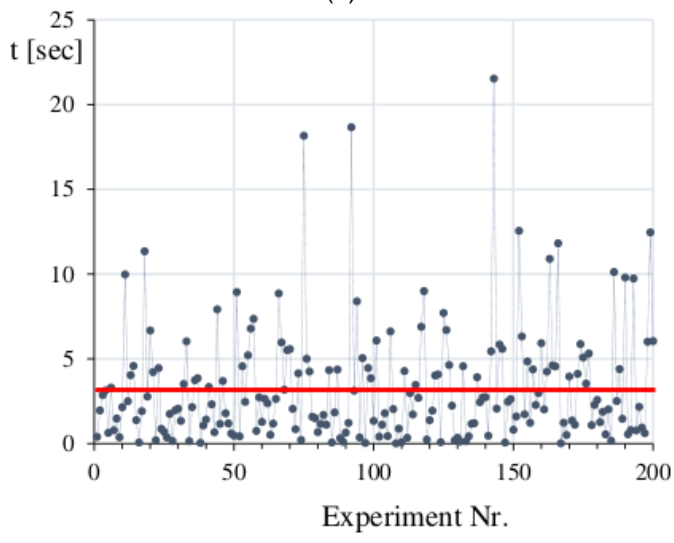

(b)

Fig. 5. Examples of results from test generation

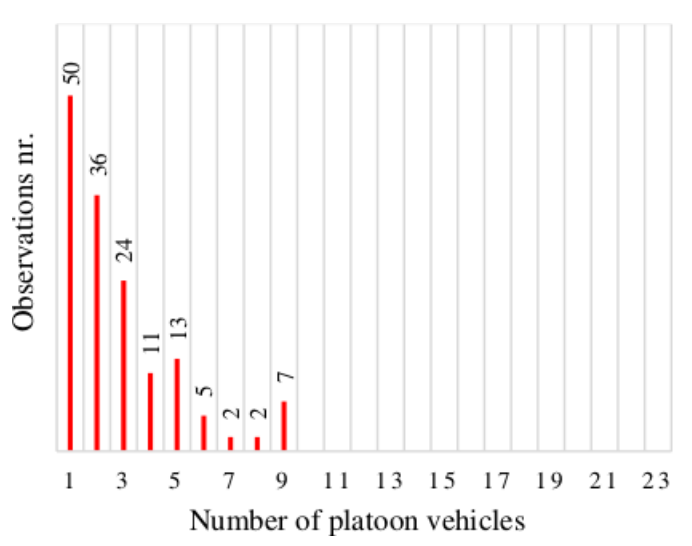

Fig. 6. Example of number distribution of platooned vehicles

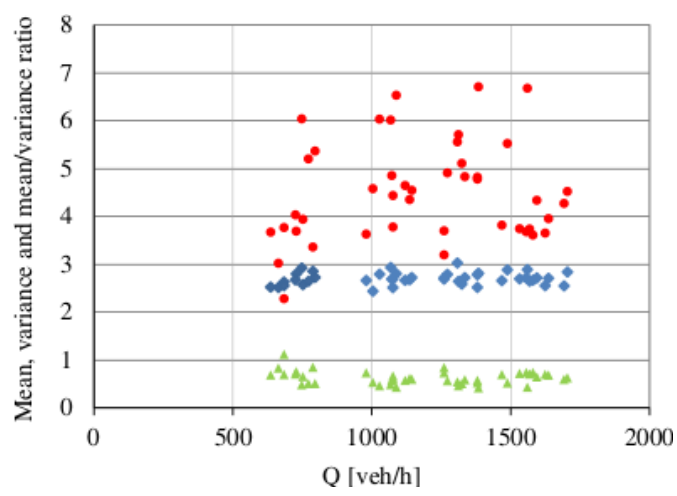

Fig. 7. Characteristics of the platoon number distribution (blue: mean; red: variance; green: mean/variance ratio) according to time headway exponential negative distribution 
Tab. 3. Characteristics of number distributions of platoons per $Q_{\text {theoretical }}=720 \mathrm{veh} / \mathrm{h}$

\begin{tabular}{|c|c|c|c|c|c|c|c|c|}
\hline \multirow{3}{*}{ Test } & \multicolumn{4}{|c|}{ Negative exponential } & \multicolumn{4}{|c|}{ Erlang } \\
\hline & \multirow{2}{*}{$Q[\mathrm{veh} / \mathrm{h}]$} & \multicolumn{3}{|c|}{ Distribution characteristics } & \multirow{2}{*}{$Q[\mathrm{veh} / \mathrm{h}]$} & \multicolumn{3}{|c|}{ Distribution characteristics } \\
\hline & & Mean & Variance & $\begin{array}{c}\text { mean/ } \\
\text { variance }\end{array}$ & & Mean & Variance & $\begin{array}{c}\text { mean/ } \\
\text { variance }\end{array}$ \\
\hline 1 & 664 & 2.515 & 3.023 & 0.832 & 728 & 2.395 & 1.975 & 1.212 \\
\hline 2 & 684 & 2.631 & 3.768 & 0.698 & 716 & 2.452 & 2.279 & 1.076 \\
\hline 3 & 684 & 2.552 & 2.281 & 1.119 & 688 & 2.457 & 3.440 & 0.714 \\
\hline 4 & 752 & 2.575 & 3.942 & 0.653 & 728 & 2.600 & 3.316 & 0.784 \\
\hline 5 & 788 & 2.855 & 3.361 & 0.849 & 728 & 2.304 & 2.881 & 0.800 \\
\hline 6 & 728 & 2.800 & 3.694 & 0.758 & 728 & 2.193 & 3.158 & 0.694 \\
\hline 7 & 748 & 2.922 & 6.041 & 0.484 & 716 & 2.325 & 3.433 & 0.677 \\
\hline 8 & 636 & 2.524 & 3.673 & 0.687 & 684 & 2.443 & 2.366 & 1.032 \\
\hline 9 & 772 & 2.644 & 5.205 & 0.508 & 688 & 2.493 & 2.930 & 0.851 \\
\hline 10 & 796 & 2.726 & 5.368 & 0.508 & 752 & 2.611 & 3.649 & 0.715 \\
\hline Mean & 725 & 2.674 & 4.036 & 0.710 & 716 & 2.427 & 2.943 & 0.856 \\
\hline
\end{tabular}

Tab. 4. Characteristics of number distributions of platoons per $Q_{\text {theoretical }}=1020 \mathrm{veh} / \mathrm{h}$

\begin{tabular}{|c|c|c|c|c|c|c|c|c|}
\hline \multirow{3}{*}{ Test } & \multicolumn{4}{|c|}{ Negative exponential } & \multicolumn{4}{|c|}{ Erlang } \\
\hline & \multirow{2}{*}{$Q[\mathrm{veh} / \mathrm{h}]$} & \multicolumn{3}{|c|}{ Distribution characteristics } & \multirow{2}{*}{$Q[\mathrm{veh} / \mathrm{h}]$} & \multicolumn{3}{|c|}{ Distribution characteristics } \\
\hline & & Mean & Variance & $\begin{array}{c}\text { mean/ } \\
\text { variance }\end{array}$ & & Mean & Variance & $\begin{array}{c}\text { mean/ } \\
\text { variance }\end{array}$ \\
\hline 1 & 1088 & 2.804 & 6.534 & 0.429 & 1068 & 2.363 & 3.126 & 0.756 \\
\hline 2 & 1076 & 2.514 & 3.780 & 0.665 & 936 & 2.629 & 4.918 & 0.535 \\
\hline 3 & 1004 & 2.437 & 4.582 & 0.532 & 1072 & 2.552 & 3.807 & 0.670 \\
\hline 4 & 1068 & 2.934 & 6.018 & 0.488 & 1028 & 2.677 & 5.210 & 0.514 \\
\hline 5 & 1136 & 2.679 & 4.353 & 0.615 & 1076 & 2.280 & 2.801 & 0.814 \\
\hline 6 & 1120 & 2.667 & 4.647 & 0.574 & 988 & 2.714 & 4.495 & 0.604 \\
\hline 7 & 1144 & 2.724 & 4.548 & 0.599 & 1072 & 2.552 & 3.807 & 0.670 \\
\hline 8 & 1028 & 2.793 & 6.034 & 0.463 & 1028 & 2.677 & 5.210 & 0.514 \\
\hline 9 & 1076 & 2.690 & 4.438 & 0.606 & 1076 & 2.280 & 2.801 & 0.814 \\
\hline 10 & 980 & 2.663 & 3.632 & 0.733 & 988 & 2.714 & 4.495 & 0.604 \\
\hline Mean & 1072 & 2.691 & 4.857 & 0.570 & 1033 & 2.544 & 4.067 & 0.649 \\
\hline
\end{tabular}

Tab. 5. Characteristics of number distributions of platoons per $Q_{\text {theoretical }}=1320 \mathrm{veh} / \mathrm{h}$

\begin{tabular}{|c|c|c|c|c|c|c|c|c|}
\hline \multirow{3}{*}{ Test } & \multicolumn{4}{|c|}{ Negative exponential } & \multicolumn{4}{|c|}{ Erlang } \\
\hline & \multirow{2}{*}{$Q[\mathrm{veh} / \mathrm{h}]$} & \multicolumn{3}{|c|}{ Distribution characteristics } & \multirow{2}{*}{$Q[\mathrm{veh} / \mathrm{h}]$} & \multicolumn{3}{|c|}{ Distribution characteristics } \\
\hline & & Mean & Variance & $\begin{array}{c}\text { mean/ } \\
\text { variance }\end{array}$ & & Mean & Variance & $\begin{array}{c}\text { mean/ } \\
\text { variance }\end{array}$ \\
\hline 1 & 1380 & 2.518 & 4.781 & 0.527 & 1332 & 2.431 & 3.615 & 0.672 \\
\hline 2 & 1468 & 2.659 & 3.817 & 0.697 & 1404 & 2.294 & 2.775 & 0.827 \\
\hline 3 & 1272 & 2.789 & 4.911 & 0.568 & 1368 & 2.591 & 3.938 & 0.658 \\
\hline 4 & 1324 & 2.586 & 5.111 & 0.506 & 1352 & 2.522 & 4.296 & 0.587 \\
\hline 5 & 1380 & 2.782 & 4.822 & 0.577 & 1332 & 2.562 & 3.597 & 0.712 \\
\hline 6 & 1384 & 2.813 & 6.711 & 0.419 & 1336 & 2.420 & 2.158 & 1.122 \\
\hline 7 & 1308 & 3.028 & 5.560 & 0.545 & 1260 & 2.582 & 3.254 & 0.794 \\
\hline 8 & 1260 & 2.716 & 3.701 & 0.734 & 1352 & 2.397 & 3.198 & 0.750 \\
\hline 9 & 1312 & 2.645 & 5.710 & 0.463 & 1316 & 2.301 & 3.381 & 0.681 \\
\hline 10 & 1260 & 2.692 & 3.198 & 0.842 & 1240 & 2.480 & 2.542 & 0.976 \\
\hline Mean & 1335 & 2.723 & 4.832 & 0.588 & 1329 & 2.458 & 3.275 & 0.778 \\
\hline
\end{tabular}


Tab. 6. Characteristics of number distributions of platoons per $Q_{\text {theoretical }}=1620 \mathrm{veh} / \mathrm{h}$

\begin{tabular}{|c|c|c|c|c|c|c|c|c|}
\hline \multirow{3}{*}{ Test } & \multicolumn{4}{|c|}{ Negative exponential } & \multicolumn{4}{|c|}{ Erlang } \\
\hline & \multirow{2}{*}{$Q[\mathrm{veh} / \mathrm{h}]$} & \multicolumn{3}{|c|}{ Distribution characteristics } & \multirow{2}{*}{$Q[\mathrm{veh} / \mathrm{h}]$} & \multicolumn{3}{|c|}{ Distribution characteristics } \\
\hline & & Mean & Variance & $\begin{array}{c}\text { mean/ } \\
\text { variance }\end{array}$ & & Mean & Variance & $\begin{array}{c}\text { mean/ } \\
\text { variance }\end{array}$ \\
\hline 1 & 1704 & 2.840 & 4.525 & 0.628 & 1640 & 2.563 & 4.386 & 0.584 \\
\hline 2 & 1556 & 2.720 & 3.682 & 0.739 & 1644 & 2.553 & 3.611 & 0.707 \\
\hline 3 & 1532 & 2.697 & 3.745 & 0.720 & 1620 & 2.396 & 2.074 & 1.155 \\
\hline 4 & 1692 & 2.548 & 4.273 & 0.596 & 1600 & 2.597 & 3.419 & 0.760 \\
\hline 5 & 1568 & 2.649 & 3.740 & 0.708 & 1596 & 2.229 & 2.885 & 0.773 \\
\hline 6 & 1636 & 2.709 & 3.955 & 0.685 & 1580 & 2.453 & 3.137 & 0.782 \\
\hline 7 & 1488 & 2.884 & 5.525 & 0.522 & 1632 & 2.534 & 3.975 & 0.637 \\
\hline 8 & 1560 & 2.889 & 6.682 & 0.432 & 1524 & 2.540 & 3.525 & 0.721 \\
\hline 9 & 1624 & 2.553 & 3.654 & 0.699 & 1604 & 2.475 & 3.916 & 0.632 \\
\hline 10 & 1580 & 2.669 & 3.611 & 0.739 & 1560 & 2.422 & 2.920 & 0.829 \\
\hline Mean & 1594 & 2.716 & 4.339 & 0.647 & 1600 & 2.476 & 3.385 & 0.758 \\
\hline
\end{tabular}

Tab. 7. Comparison between results obtained from the model and from measurement campaigns on operational roads

\begin{tabular}{|c|c|c|c|c|}
\hline & Observed value & $\begin{array}{l}\text { Results from } \\
\text { measurement } \\
\text { campaigns }\end{array}$ & $\begin{array}{c}\text { Results from } \\
\text { application of the } \\
\text { model }\end{array}$ & Percent deviation \\
\hline & $\begin{array}{c}\text { Flow rate mean } \\
\text { [veh/20 sec] }\end{array}$ & 2.389 & 2.389 & - \\
\hline \multirow[t]{4}{*}{ Test 1} & Flow rate variance & 5.830 & 4.927 & $-15.49 \%$ \\
\hline & $\begin{array}{c}\text { Platoon number } \\
\text { mean [veh] }\end{array}$ & 3.024 & 2.876 & $-4.89 \%$ \\
\hline & $\begin{array}{c}\text { Platoon number } \\
\text { variance }\end{array}$ & 6.348 & 7.323 & $15.36 \%$ \\
\hline & $\begin{array}{c}\text { Flow rate mean } \\
\text { [veh/20 sec] }\end{array}$ & 2.878 & 2.878 & - \\
\hline \multirow[t]{4}{*}{ Test 2} & Flow rate variance & 7.899 & 6.944 & $-12.09 \%$ \\
\hline & $\begin{array}{c}\text { Platoon number } \\
\text { mean [veh] }\end{array}$ & 2.981 & 2.793 & $-6.31 \%$ \\
\hline & $\begin{array}{c}\text { Platoon number } \\
\text { variance }\end{array}$ & 5.328 & 4.992 & $-6.31 \%$ \\
\hline & $\begin{array}{c}\text { Flow rate mean } \\
\text { [veh/20 sec] }\end{array}$ & 2.111 & 2.111 & - \\
\hline \multirow[t]{4}{*}{ Test 3} & Flow rate variance & 3.600 & 3.356 & $-6.78 \%$ \\
\hline & $\begin{array}{c}\text { Platoon number } \\
\text { mean [veh] }\end{array}$ & 2.376 & 2.498 & $5.13 \%$ \\
\hline & $\begin{array}{c}\text { Platoon number } \\
\text { variance }\end{array}$ & 5.927 & 6.782 & $14.43 \%$ \\
\hline & $\begin{array}{c}\text { Flow rate mean } \\
\text { [veh/20 sec] }\end{array}$ & 1.550 & 1.550 & - \\
\hline \multirow[t]{4}{*}{ Test 4} & Flow rate variance & 3.110 & 3.593 & $15.53 \%$ \\
\hline & $\begin{array}{c}\text { Platoon number } \\
\text { mean [veh] }\end{array}$ & 2.668 & 2.753 & $3.19 \%$ \\
\hline & $\begin{array}{c}\text { Platoon number } \\
\text { variance }\end{array}$ & 4.680 & 4.901 & $4.72 \%$ \\
\hline & $\begin{array}{c}\text { Flow rate mean } \\
\text { [veh/20 sec] }\end{array}$ & 1.083 & 1.083 & - \\
\hline \multirow[t]{3}{*}{ Test 5} & Flow rate variance & 0.762 & 1.004 & $31.76 \%$ \\
\hline & $\begin{array}{c}\text { Platoon number } \\
\text { mean [veh] }\end{array}$ & 1.982 & 2.096 & $5.75 \%$ \\
\hline & $\begin{array}{c}\text { Platoon number } \\
\text { variance }\end{array}$ & 3.925 & 4.703 & $19.82 \%$ \\
\hline
\end{tabular}




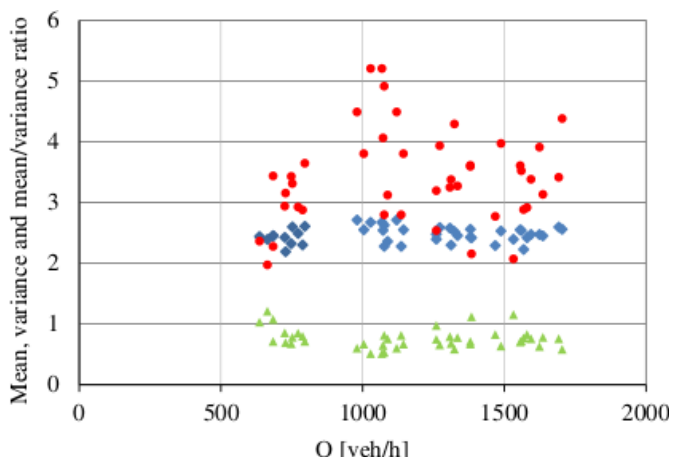

Fig. 8. Characteristics of the platoon number distribution (blue: mean; red: variance; green: mean/variance ratio) according to time headway Erlang distribution

\section{Generation of traffic process realizations}

The algorithm can also generate realizations of the random function $Q(t)$ "traffic flow $Q$ on a road cross-section in function of time $t$ ". In general, a random function is defined as a function which, after a proof, can take a concrete form; this form is called random variable realization. Unlike ordinary random variables, random functions represent sizes which vary continuously during the proof [17].

In this case the random function independent variable is as usual represented by the time $t$. Here, the random variable $Q(t)$ is a continuous function in the sample interval.

A realization cannot strictly be represented in a continuous form, but rather through discrete values with sufficiently close time intervals which can guarantee a very precise image of its variations. It is goes without saying that a random function realization becomes a (discrete) random variable for a set $t$ value. Therefore, the random variable $Q(t)$ realizations were represented by means of the values which are assumed by such a variable in set time instants $t$, corresponding to instants where a single vehicle passage is simulated.

More in detail, for time instant $t=t^{*}$ the flow $Q\left(t^{*}\right)$ was obtained by means of time headways generated by the algorithm as follows: $Q\left(t^{*}\right)$ is equal to the number $n\left(t^{*}\right)$ of vehicles passing from instant 0 to instant $t^{*}$, divided by time $t^{*}$ :

$$
Q\left(t^{*}\right)=\frac{n\left(t^{*}\right)}{t^{*}}
$$

seen that

$$
t^{*}=\sum_{i=0}^{n} t_{i}
$$

with $t_{i}$ : time headway of $\mathrm{i}$-th vehicle.

In other words, $t^{*}$ is the sum of time headways of $n$ vehicles in question.

This procedure allowed to obtain the realization families for all the tests carried out by using the calculus algorithm, with regard to the same classes indicated in paragraph 5 (that is to say, with flow rates equal approximately to $720,1020,1320$, $1620 \mathrm{veh} / \mathrm{h})$.

The graphical representation of some realization families is shown in Fig. 9 and Fig. 10 for time headways consistent with the negative exponential and Erlang distributions respectively. We observed that in all realizations the functions stabilized around the mean flow rate value, previously set as input datum in the calculus algorithm, after a first time interval in which the flow rate $Q$ oscillated between very high values and values close to zero.

This can be explained by the fact that in the first part of the observation interval the reduced number of passing vehicles could not be considered as representative of the phenomenon yet. On the other hand, when time $t$ (and therefore, the observed vehicle number) increased, the sample turned out to be more representative and consequently the observed flow rate value tended to the mean value. Finally, we also estimated the mathematical hope (meant as the non random function which is equal to the mathematical hope of the corresponding random function section for each value of the argument $t$ ) and the variance (meant as the non random function whose value is equal to the variance of the corresponding random function section for each $t$ ) for the realization families.

The values observed for these characteristic functions (mathematical hope and variance), obtained in a discrete form for points with set intervals $\Delta t$ confirmed what expected on the basis of the steady state flow hypothesis, or in other words that the mathematical hope is very close to a constant function with a value equal to the mean flow rate and variance is, in all cases, a decreasing monotonous function. The latter observation especially indicates the reduction in the dispersion of random function realizations with regard to the mean when time $t$ (and therefore the number of passing vehicle) increases.

\section{Conclusions}

This research provides a further scientific contribution to the platoon size distribution analysis in steady-state flow conditions and on highway facilities with uninterrupted flow, based on the generalized probability law (Pearson type III law) and on its derived laws after properly assigning coefficient values in its formula.

Notably, we examined and applied two derived laws, the negative exponential and Erlang distributions, both used to calculate the time headway between vehicles of a set traffic flow. We then analysed vehicle platoon distribution within a traffic stream.

For the purpose of this study, consistently with the convention followed in numerous scientific studies, by "platoon" we meant the set of vehicles - with the exception of the last in the file - presenting vehicle headways lower than the mean headway $\bar{t}$, whose number $N$ is higher than or equal to 2 and transiting one after the other along the observational road cross-section.

In order to analyse quite a sufficient number of data, which can hardly be collected from empirical traffic surveys, we designed an appropriate algorithm to randomly generate tests which simulate a steady state-regime flow and provide the generated vehicle with time headways. We tested such an algorithm to verify its reliability and strength by comparing the out- 


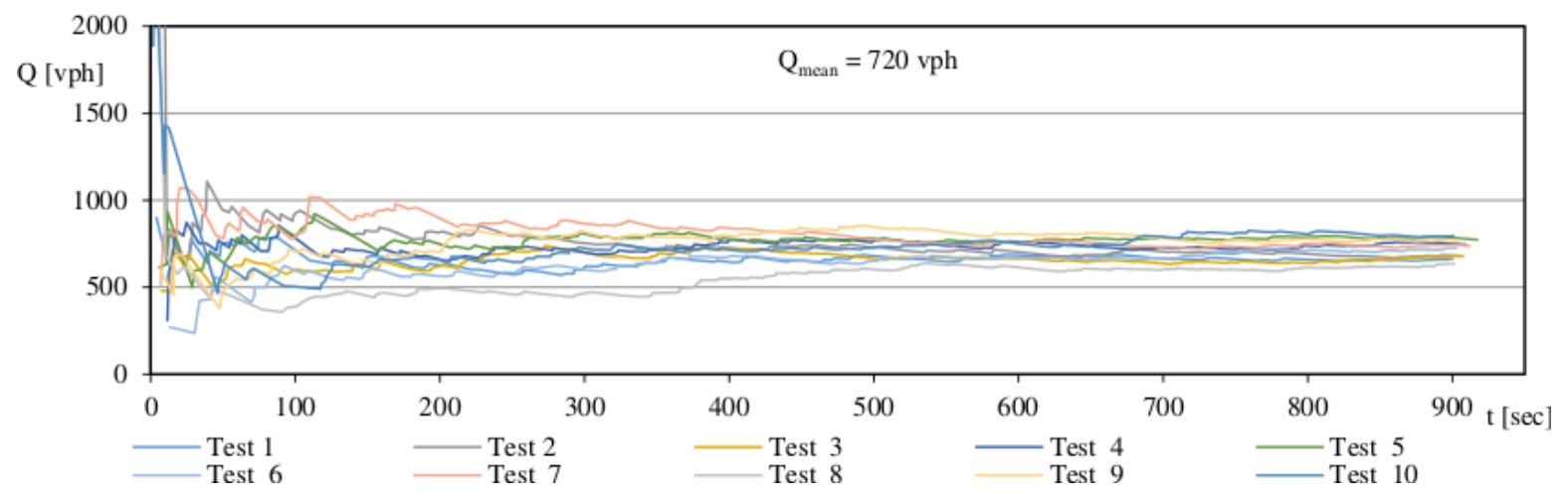

(a)

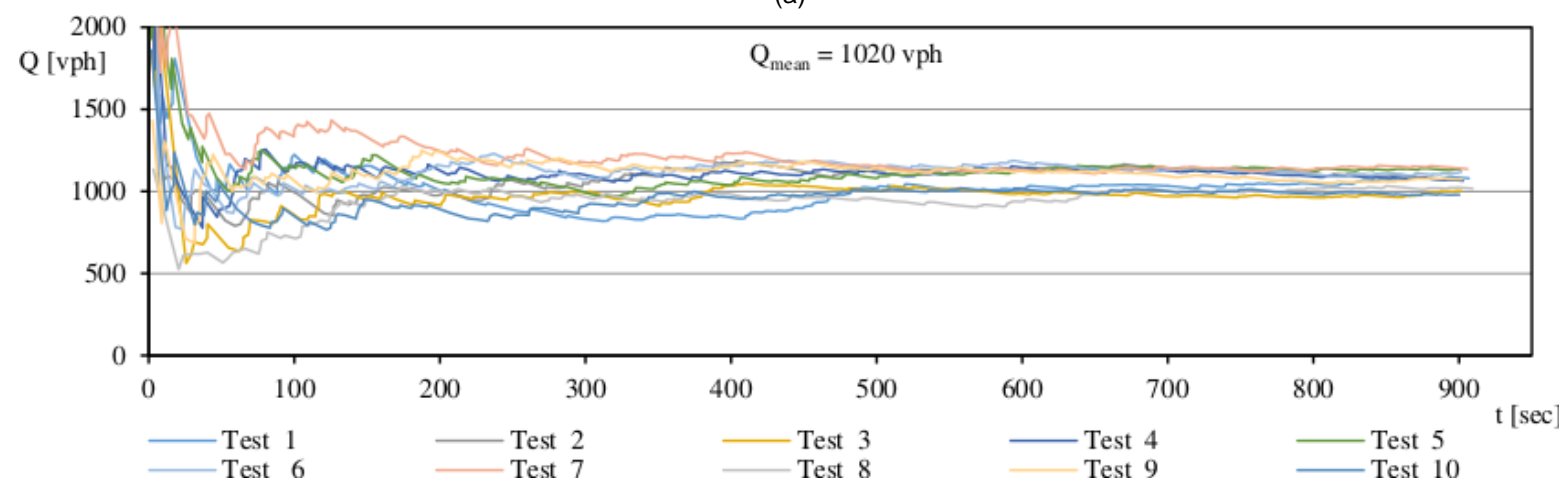

(b)

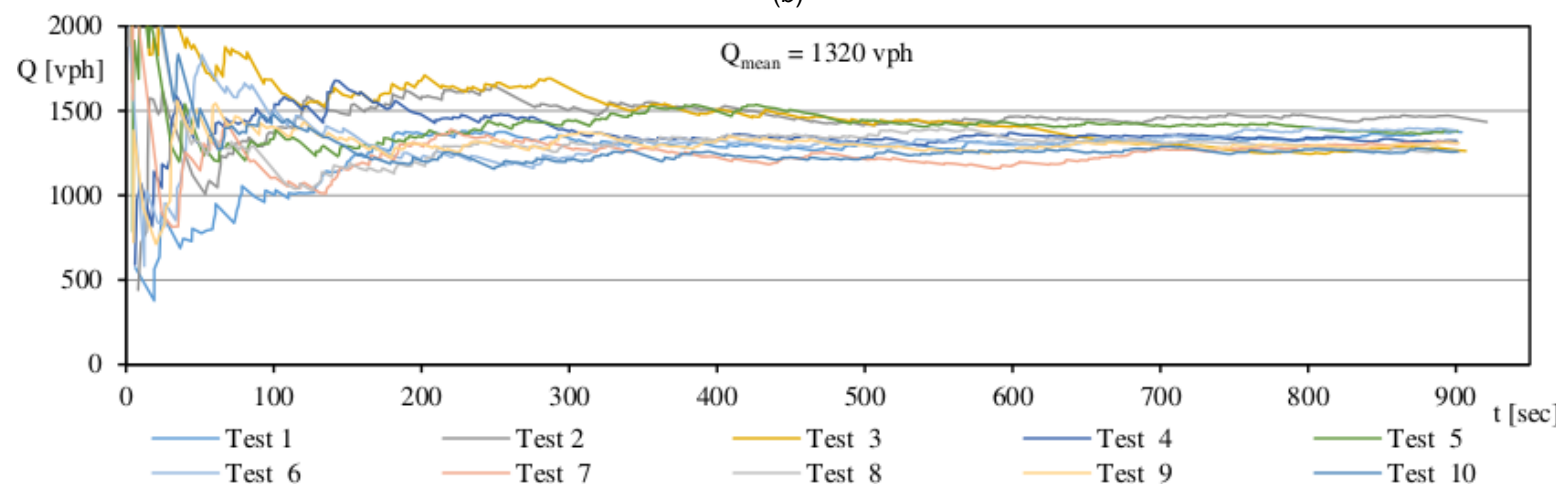

(c)

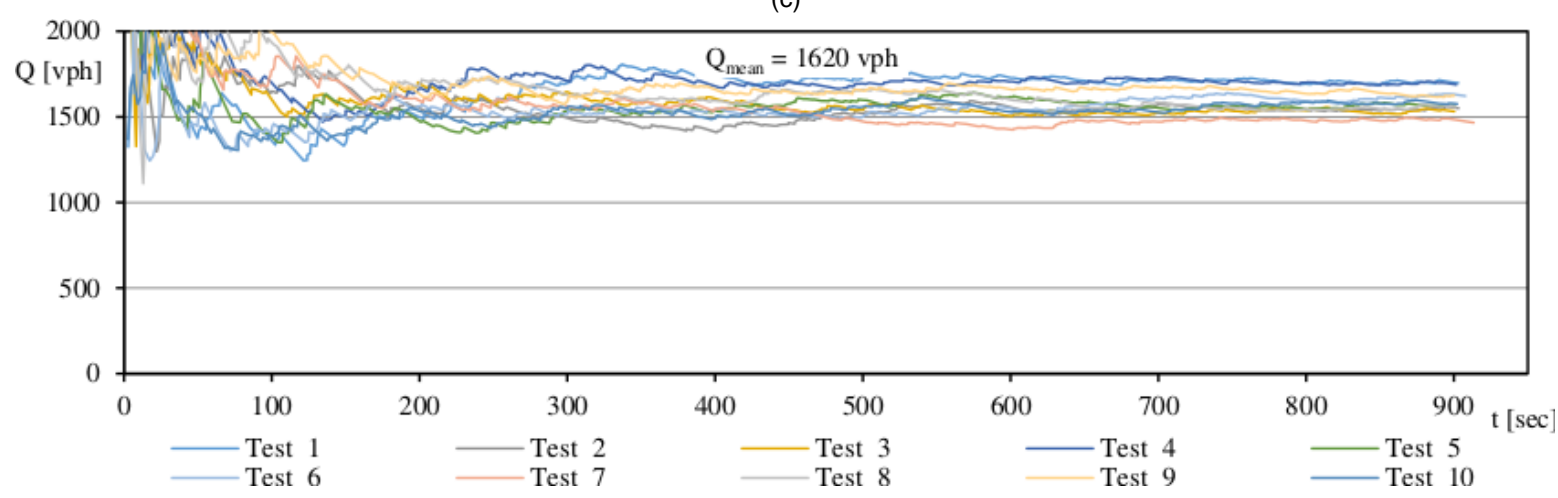

(d)

Fig. 9. Examples of families of random variable $Q(t)$ realizations - headways with a negative exponential distribution 


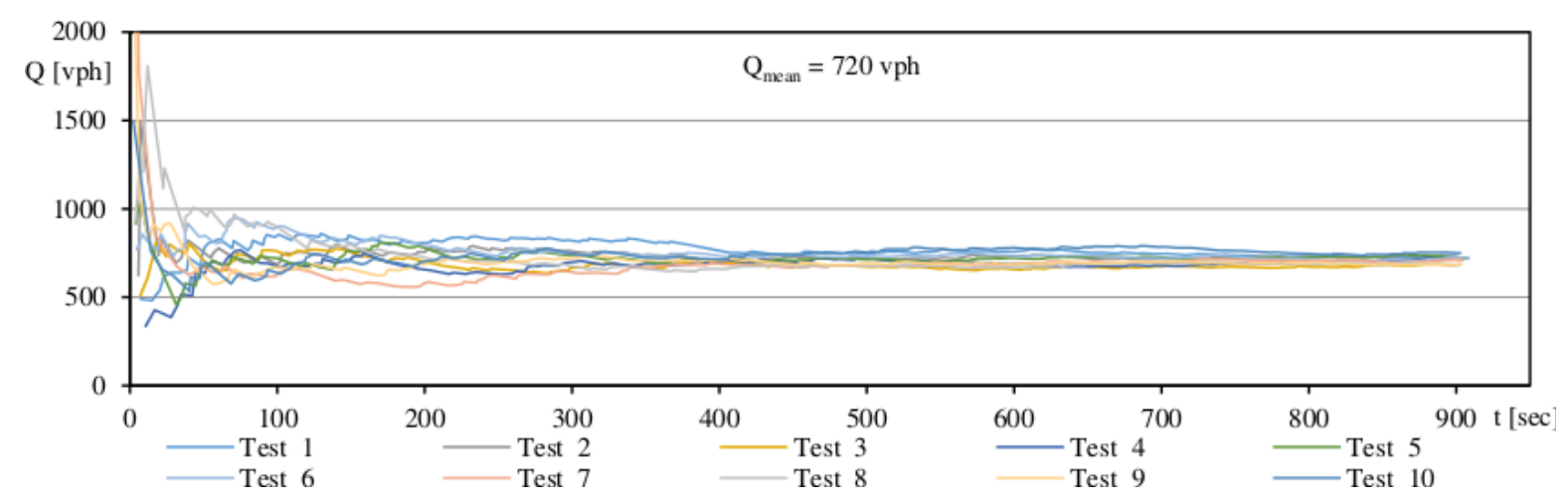

(a)

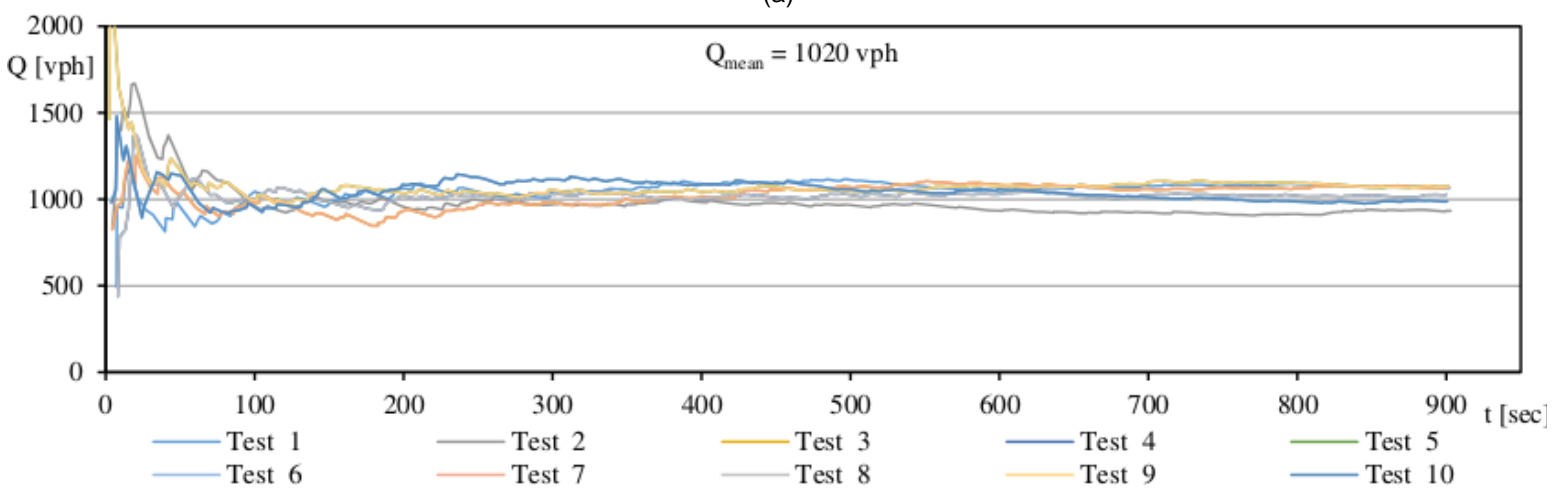

(b)

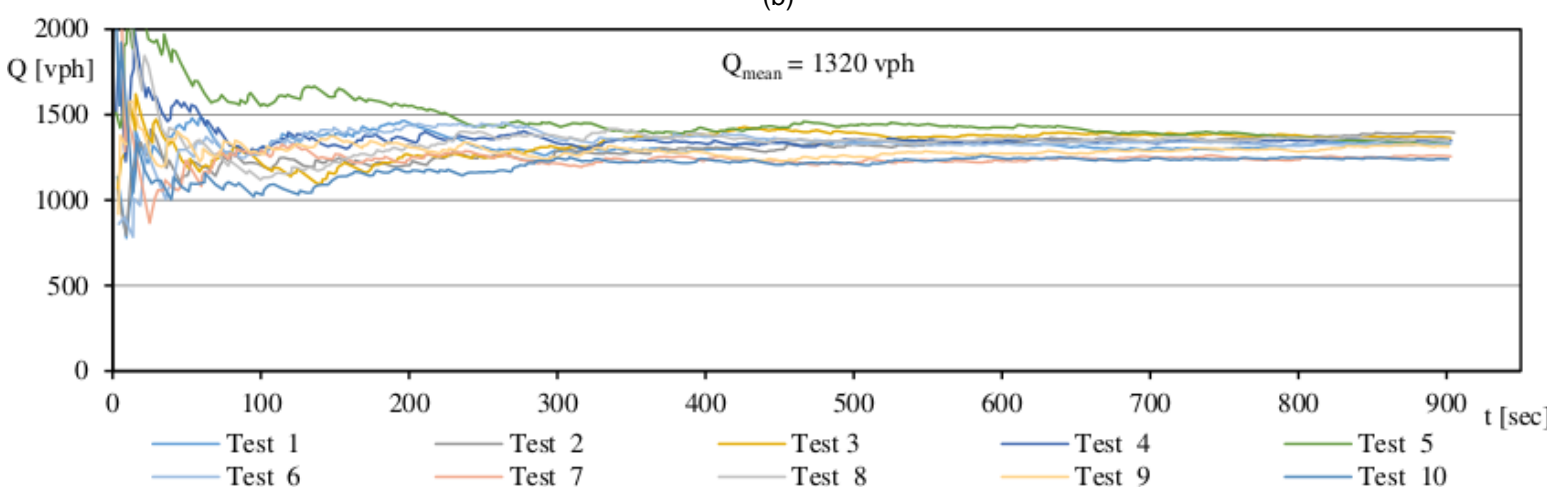

(c)

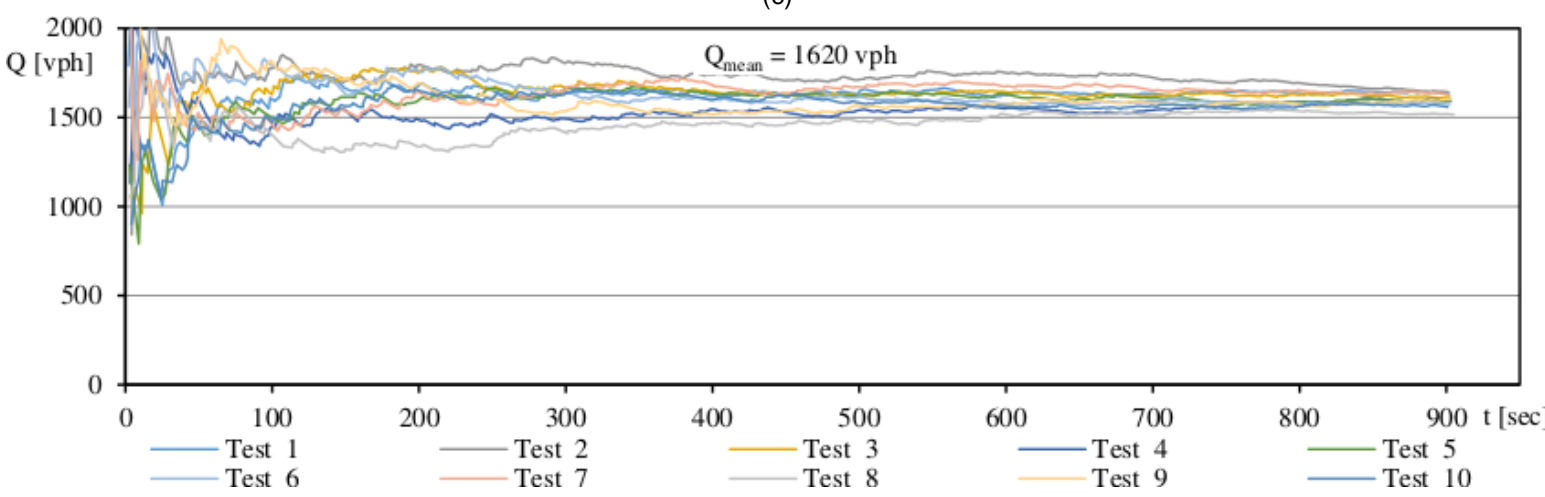

(d)

Fig. 10. Examples of families of random variable $Q(t)$ realizations - headways with Erlang distribution 
put with the empirical data observed during some measurement campaigns of time headways on Italian roads into operation. Results obtained from some of the tests carried out are shown.

Then, we used the algorithm to generate an adequate number of tests and to analyse platooned vehicle distribution and number within a traffic stream in a steady state regime.

The results of several tests allow to reach the conclusion that the platoon-forming vehicle number mean assumes a value ranging between 2 and 3 in any case. Moreover, there is no correlation between the flow rate value and the mean itself. Similar observation can be made for variance which is comprised between 2 and 7 except for some tests and for the ratio mean/variance always lower than 1.25.

The algorithm can also generate realizations of the random function $Q(t)$ "traffic flow $Q$ on a road cross-section in function of time $t$ ". We calculated the mathematical hope and variance for families of realizations. The values obtained for the characteristic functions (mathematical hope and variance) in a discrete manner (at set time intervals) confirmed what expected on the basis of the flow stationarity hypothesis: the mathematical hope assumes values close to a constant function with a value equal to the mean flow rate and, in any case, the variance is a decreasing monotonous function. With direct reference to variance, when time $t$ increases (and consequently the passing vehicle number), the dispersion of random function realizations is more reduced than the mean.

\section{References}

1 Appert-Rolland C, Santen L, Accidents in Platoons of Vehicles: Schadschneider A, Poschel T, Kuhne R, Schreckenberg M, Wolf D E (eds.), Traffic and Granular Flow'05, Springer; Berlin, Germany, 2007, pp. 623-631.

2 Baras J, Dorsey A, Levine W, Estimation of traffic platoon structure from headway statistics, IEEE Transactions on Automatic Control, 24(4), (1979), 553-559, DOI 10.1109/TAC.1979.1102118

3 Cal y Mayor Reyes Spindola R, Cardenas Grisales J, Ingenieria de transito, 7th, Alfaomega, 1994.

4 Dell'Acqua G, De Luca M, Mauro R, Lamberti R, Motorway Speed Management in Southern Italy, Procedia - Social and Behavioral Sciences, 20, (2011), 49-58, DOI 10.1016/j.sbspro.2011.08.010

5 Fi I, Galuska J, Recommendations for new capacity values on freeways, Periodica Polytechnica Civil Engineering, 54(2), (2010), 127-136, DOI 10.3311/pp.ci.2010-2.08

6 Fi I, Galuska J, Traffic analysis of two-lane highways, Periodica Polytechnica Civil Engineering, 55(2), (2011), 169-176, DOI 10.3311/pp.ci.20112.09

7 Gartner N, Messer C J, Rathi A K, Traffic flow theory. A state- of-theart report, 1th, Committee on Traffic Flow Theory and Characteristics, TRB; Washington D.C., 2001.

8 Geistefeldt J, Brilon W, A Comparative Assessment of Stochastic Capacity Estimation Methods, In: Lam W H K, Wong S C, Lo H K (eds.), Transportation and Traffic Theory 2009: Golden Jubilee, Springer; Berlin, Germany, 2009, pp. 583-602.

9 Jiang Y, Li S, Shamo D, Development of Vehicle Platoon Distribution Models and Simulation of Platoon Movements on Indian Rural Corridors, Publication FHWA/IN/JTRP-2002/23. Joint Transportation Research Program; Indiana Department of Transportation and Purdue University, West Lafayette, Indiana, 2003, DOI $10.5703 / 1288284313195$
10 Luspay T, Kulcsár B, Varga I, Bokor J, Parameter dependent freeway modelling, Periodica Polytechnica Transportation Engineering, 36(1-2), (2008), 61-67, DOI 10.3311/pp.tr.2008-1-2.12

11 May A D, Traffic flow fundamentals, NY: Prentice Hall; New Jersey, 1990.

12 Mauro R, Branco F, Update on the Statistical Analysis of Traffic Countings on Two-Lane Rural Highways, Modern Applied Science, 7(6), (2013), 67-80, DOI $10.5539 /$ mas.v7n6p67

13 Mauro R, Giuffrè O, Granà A, Speed Stochastic Processes and Freeway Reliability Estimation: Evidence from the A22 Freeway, Italy, Journal of Transportation Engineering, 139(12), (2013), 1244-1256, DOI 10.1061/(ASCE)TE.1943-5436.0000599

14 Mauro R, Branco F, Two Vehicular Headways Time Dichotomic Models, Modern Applied Science, 6(12), (2012), 1-12, DOI 10.5539/mas.v6n12p1

15 Ramezani H, Benekohal R F, Avrenli K A, Statistical distribution for inter platoon gaps, intra-platoon headways and platoon size using field data from highway bottlenecks, In: Summer meeting of the TFTC Committee and Second workshop of the Multitude COST action; Annecy, France, 2010.

16 Shiomi Y, Yoshii T, Kitamura R, Platoon-Based Traffic Flow Model for Estimating Breakdown Probability at Single-Lane Expressway Bottlenecks, In:, Vol. 17, 2011, pp. 591-610, DOI 10.1016/j.sbspro.2011.04.533 Papers selected for the 19th International Symposium on Transportation and Traffic Theory.

17 Wentzel E, Ovcharov L, Applied problems in probability theory, Mir Publishers; Moscow, 1986. 